Research Article

\title{
Prediction Model of Build Rate of Push-the-Bit Rotary Steerable System
}

\author{
Meishan Wang, ${ }^{1}$ Xiaojun Li, ${ }^{2,3}$ Ge Wang, ${ }^{4}$ Wenjun Huang $\left(\mathbb{D},{ }^{4}\right.$ Yongtao Fan, ${ }^{3,5}$ Wei Luo, ${ }^{2,3}$ \\ Jiange Zhang, ${ }^{3,6}$ Junfeng Zhang, ${ }^{2,3}$ and Xiaolei Shi ${ }^{4}$ \\ ${ }^{1}$ Science and Technology Information Department, CNPC Oilfield Service Co., Ltd., Beijing 102206, China \\ ${ }^{2}$ Engineering Technology Research Institute, CNPC XIBU Drilling Engineering Co., Ltd., Urumqi 830000, China \\ ${ }^{3}$ Well Logging Technology Research Institute, China Petroleum Logging Co., Ltd., Beijing 102206, China \\ ${ }^{4}$ School of Petroleum Engineering, China University of Petroleum-Beijing, Beijing 102249, China \\ ${ }^{5}$ Engineering Technology Research Institute, CNPC BOHAI Drilling Engineering Co., Ltd., Tianjin 300457, China \\ ${ }^{6}$ Logging While Drilling Center, China Petroleum Logging Co., Ltd., Xi'an 710000, China
}

Correspondence should be addressed to Wenjun Huang; huangwenjun1986@126.com

Received 4 February 2020; Accepted 5 May 2020; Published 22 May 2020

Academic Editor: Francesc Pozo

Copyright ( $\odot 2020$ Meishan Wang et al. This is an open access article distributed under the Creative Commons Attribution License, which permits unrestricted use, distribution, and reproduction in any medium, provided the original work is properly cited.

The accurate prediction of build rate of rotary steerable system (RSS) is the prerequisite for the precise control of wellbore trajectory and optimal design of the steerable tool. Push-the-bit RSS refers to a steerable drilling system that controls well trajectory through adjustment of stretch length of pushing block. Based on this feature, a three-dimensional mechanical model of push-the-bit RSS is established on the basis of the conventional steerable tool mechanical model while considering pushing force. Meanwhile, the effects of bit cutting performance and formation anisotropy are considered. Next, the lateral force method, inclination trend angle method, and ultimate build rate method based on zero lateral force are analyzed and a new ultimate build rate method based on zero lateral drilling penetration is built. A conversion coefficient is introduced to reflect the ratio of actual build rate to theoretical build rate. At last, the new model is applied to a case study. The new method is superior to other previous methods, because the effects of bit-rock interaction and actual drilling process are sufficiently considered. The influence mechanism of build rate includes "leverage effect," "pendulum effect," and "pushing effect." The build rate of steerable tool is a comprehensive outcome of various effects, of which the "pushing effect" plays a dominant role. With the increase of weight on bit (WOB), the proportion of "leverage effect" increases, while the proportion of "pushing effect" decreases, and therefore the build rate decreases.

\section{Introduction}

It is usually difficult to accurately control the trajectory in directional drilling, especially when drilling in low-porosity and low-permeability horizontal thin formations. When the inclination angle of stratum is changeable, the wellbore trajectory may appear to tilt up or down in this situation. Then, it is rather difficult to control trajectory in the optimum location of reservoirs $[1,2]$. Recently, RSS is the most effective tool in accurate trajectory control.

Push-the-bit RSS utilizes reaction force generating from the interaction between pushing block and wellbore surface to change the lateral force on drill bit, and then lateral drilling penetration is changed. Thus, well trajectory can be controlled through the adjustment of stretch length of pushing block. Compared with other conventional steerable tools, RSS have many advantages: (1) drilling string is rotating constantly and lower friction is achieved; (2) wellbore trajectory can be adjusted in real time; (3) higher drilling penetration is obtained [3-8].

However, sometimes high build rates cannot be achieved and build rates are not stable in actual drilling process. To accurately control trajectory, some problems should be solved in advance, such as accurate prediction of the build 
rate, structure optimization design of bottom-hole assembly (BHA), and optimization design of drilling parameters in directional drilling.

In order to exert the performance of push-the-bit RSS, the features of its build rate need to be fully understood. Karisson [9] used a geometric method to calculate the build rate of RSS with a bend in the external housing during the drilling process and proposed a "circle determined by three points" method. However, this method is not sufficient to consider the influence of drilling parameters and the stiffness of drilling tools; thus, the theoretical result is not equipped with actual situations. Bradley et al. [10] adopted a mechanical method to analyze the motion of RSS in the wellbore and used the statics to study the combination of the steerable tool to calculate the mechanical force at the bit, and the angle between the resultant line of action and the axis of the wellbore acts as RSS to make the build-rate prediction. This method takes into account the stiffness of tools and drilling parameters; it can accurately predict the build rate than the "circle determined by three points" method. However, it does not consider the lateral cutting capability of the drill bit, formation factors, and so on $[11,12]$, so this method is not suitable for field applications.

Xia et al. [13] proposed a balanced trend method to predict the build rate of the bottom-hole assembly (BHA), which was believed that the build rate of BHA should be equal to the dog leg severity (DLS) when the drilling trend angle is zero. This method is based on the analysis of the mechanical model of $\mathrm{BHA}$, the model of the interaction between the bit and formation, and the evaluation index of the tendency at drilling [14-16]. It overcomes the shortcoming to the mechanical method, but the results can not directly reflect the magnitude of the build rate of tools. Su [17] proposed the limit curvature method to calculate the deviation ability of rotary-steerable drilling systems. He asserted that when the lateral force at the bit is equal to zero or tending to be zero, the corresponding DLS is representative of the limit curvature of drilling tools, which can be directly to characterize the build rate of drilling tools. Nevertheless, this method is restricted to two-dimensional models and it also ignores the impact of the drilling process.

To overcome the shortcomings in previous studies, a three-dimensional prediction model of build rate based on zero lateral drilling penetration criterion is presented, which is an extension of previous two-dimensional models. In the new model, the influence of the push force direction for the build rate is further considered. The new model is compared with other previous models including lateral force method and inclination trend angle method and is applied to structure optimization design of RSS in a case study.

\section{Trajectory Prediction Model}

2.1. Mechanical Model of BHA. Considering the overall force model of BHA, it is assumed that the wellbore trajectory of $\mathrm{BHA}$ is a spatial curve, and the projection on the inclination plane and azimuth plane of this spatial curve is a curve, as shown in Figure 1. The black curve represents the spatial curve of wellbore trajectory of BHA; the blue and red curves are the projections on the inclination plane $\alpha$ and azimuth plane $\phi$ of this curve. It is noted that the intersection of the $\alpha$ plane and the $\phi$ plane is the direction of the drill bit axis $z$. The axial load distribution of BHA can be projected to the $z$ axis to simplify the calculation of the sophistication at the axial load.

Push-the-bit RSS can be deemed as a series of beams $[18,19]$. Considering the projection on the $\alpha$ plane and the $\phi$ plane is a realistic trajectory of $\mathrm{BHA}$, and thus, the governing equation of BHA on the $\alpha$ plane and the $\phi$ plane can be obtained. However, this projection has some difference, which is a difference between the deflection $u$ and the line weight $q$ of the rotary steerable bottom-hole assembly (RSBHA) on the inclination plane and azimuth plane. Therefore, let $k=1$ represent the case of the $\alpha$ plane and $k=2$ represent the case of the $\phi$ plane. The governing equation for its deformation is as follows:

$$
\frac{\mathrm{d}^{4} u_{k}}{\mathrm{~d} s^{4}}+\frac{\mathrm{d}}{\mathrm{d} s}\left(\frac{F}{\mathrm{EI}} \frac{\mathrm{d} u_{k}}{\mathrm{~d} s}\right)=q_{k}
$$

In Equation (1), the $\alpha$ plane is vertically distributed in space, so the direction of the tubular weight $q$ is always in the $\alpha$ plane all the time. Since the $\phi$ plane is perpendicular to the $\alpha$ plane, the projection of the tubular weight $q$ on the $\phi$ plane is equal to 0 from beginning to end. In a word, the influence of its gravity effect is not considered.

Generally, all nodes of the BHA may contact the borehole during the drilling process, which will cause the overall stress state of the tubular string to change. In order that the movement of the RSBHA in down-hole drilling can be studied preferably, the state of motion at this time is shown in Figure 2. Take the possible contact position of the bit, the offset mechanism, each span stabilizer, the drilling string, and the wellbore as nodes, which can be applied to divide each of the units in the RSBHA.

In the process of directional drilling, it is vital that the gravity effect has impact on the build rate of the push-the-bit RSS. So the assumption that the RSBHA touches the lower surface of wellbore at the upper tangential contact point is adopted. According to Figure 2, the deflection function in the $j$ node of the RSS can be expressed as

$$
u_{k, j}(s)=\sum_{i=1}^{5} C_{k, i, j} s^{5-i}, \quad(j=1,2, \ldots, N+1) .
$$

In Equation(2), $C_{k, i, j}$ is the coefficient to be determined and $N$ represents the myriad of nodes on the BHA. In the actual drilling process, the variation of the contact position between each node and the borehole is unknown. In order to discover some regularity, the continuous and boundary conditions may occur at each node which need to be analyzed.

Since the displacement and bending moment at the bit value are 0 , the boundary conditions at the bit can be expressed as 


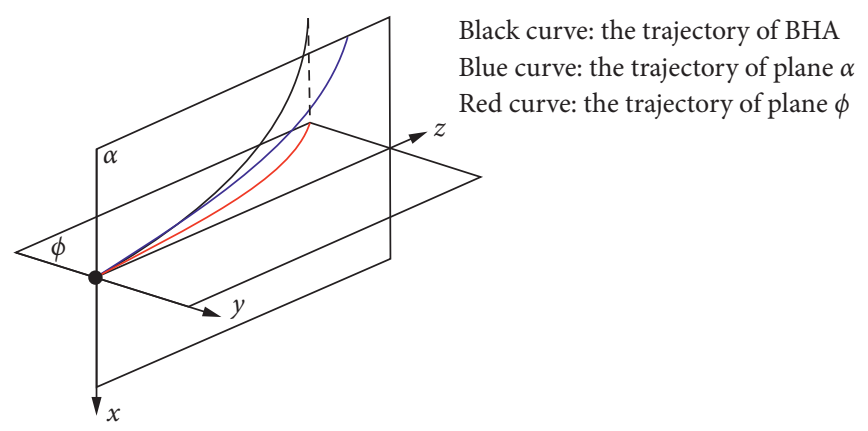

FIGURE 1: Wellbore trajectory curve projection.

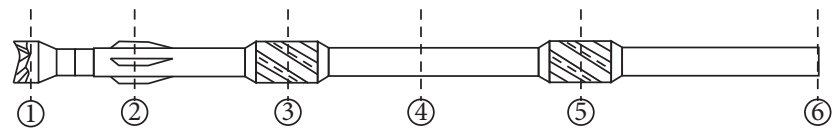

FIgURE 2: Push-the-bit RSS overall schematic.

$$
u_{k, 1}(0)=u_{k, 1}^{\prime \prime}(0)=0 \text {. }
$$

The boundary conditions at the upper tangential contact point are as follows:

$$
\left\{\begin{array}{l}
u_{k, N+1}\left(L_{k, N+1}\right)=\left(\frac{1}{\kappa_{k}}+\lambda_{k} \cdot r_{N+1}\right) \cos \psi_{k, N+1}-\frac{1}{\kappa_{k}}, \\
u_{k, N+1}^{\prime}\left(L_{k, N+1}\right)=-\left(1+\lambda_{k} \cdot r_{N+1} \cdot \kappa_{k}\right) \sin \psi_{k, N+1}, \\
u_{k, N+1}^{\prime \prime}\left(L_{k, N+1}\right)=-\left(1+\lambda_{k} \cdot r_{N+1} \cdot \kappa_{k}\right) \cos \psi_{k, N+1} \cdot \kappa_{k} .
\end{array}\right.
$$

In Equation (4), $r_{N+1}$ represents the apparent radius of the borehole at upper tangential contact point and $\lambda_{k}$ is the dimensionless coefficient. If the length $L_{k, N+1}$ of upper tangential contact point in the actual trajectory is projected to the $\alpha$ plane, the dimensionless coefficient $\lambda_{1}$ value is 1 . If the projection is to the azimuth plane, because it is an inclined straight plane, the centering effect produced by the projection needs to be considered, and the dimensionless coefficient $\lambda_{2}$ value is 0 .

If the joint is in contact with the borehole, the continuous conditions of displacement, slope, and bending moment at the joint are as follows:

$$
\left\{\begin{array}{l}
u_{k, j}\left(L_{k, j}\right)=u_{k, j+1}(0)=\left(\frac{1}{\kappa_{k}}+\mu_{k, j} \cdot r_{j}\right) \cos \psi_{k, j}-\frac{1}{\kappa_{k}} \\
u_{k, j}^{\prime}\left(L_{k, j}\right)=u_{k, j+1}^{\prime}(0) \\
\mathrm{EI}_{j} u_{k, j}^{\prime \prime}\left(L_{k, j}\right)=\mathrm{EI}_{j+1} u_{k, j+1}^{\prime \prime}(0) .
\end{array}\right.
$$

Generally, the contact point of the borehole would consist of an offset mechanism and each span stabilizer. The condition where the tubular string contacts the borehole is relatively small. The length of the RSBHA is much longer than the contact length between each node and the borehole.
Therefore, the calculation sophistication of the surface contact is simplified by considering the point contact between the node and the borehole. In order not to lose the generality, the change of the contact condition of each node with the borehole can be expressed. According to the dimensionless coefficient $\mu_{k, j}$ in the $j$ node, if contact with upper wellbore $\mu_{k, j}=-1$, contact with lower wellbore $\mu_{k, j}=0$.

When the actual drilling encounters heterogeneous formations, the well diameter may be enlarged, and the RSBHA may also be worn. These conditions may cause some nodes to be out of contact with the borehole. To handle this condition, the pushing block, stabilizer, and tubular string can be treated as a shear force. In this way, the pushing block can be distributed at each node, and the structure design of the RSBHA can be optimized. The continuous conditions of displacement, slope, bending moment, and shear force without contacting the borehole are as follows:

$$
\left\{\begin{array}{l}
u_{k, j}\left(L_{k, j}\right)=u_{k, j+1}(0), \\
u_{k, j}^{\prime}\left(L_{k, j}\right)=u_{k, j+1}^{\prime}(0), \\
\operatorname{EI}_{j} u_{k, j}^{\prime \prime}\left(L_{k, j}\right)=\mathrm{EI}_{j+1} u_{k, j+1}^{\prime \prime}(0), \\
\operatorname{EI}_{j} u_{k, j}^{\prime \prime}\left(L_{k, j}\right)-\mathrm{EI}_{j+1} u_{k, j+1}^{\prime \prime}(0)=F_{k, p}(0) .
\end{array}\right.
$$

In Equation (6), $F_{k, p}$ is the component of the push force projected on the $\alpha$ and $\phi$ planes, respectively. In terms of any $j$ node, if $F_{k, p} \neq 0$, it means that the reacting force plays a function to the wellbore at offset mechanism of the RSBHA. If the driving piston of the pushing block does not contact the wellbore or make false contact with the wellbore, the offset mechanism can be processed together with the stabilizer and tubular string that have not contacted the wellbore. Therefore, $F_{k, p}=0$.

In order to satisfy Equations (1) and (3) to Equation (6), it is necessary to utilize the subdomain method [18] to eliminate the internal residual value of Equation (2). This paper uses the subdomain method to obtain the residual equation: 


$$
\int_{s=0}^{L_{j}} \varepsilon_{k, j}=0 .
$$

In Equation (7), $\varepsilon_{k, j}$ is the internal residual value. For the $\alpha$ plane, it can be demonstrated that there are 5 parameters to be determined for each span deformation, and the length from the upper tangential contact point to the stabilizer needs 1 parameter to be confirmed, so there are $5 \mathrm{~N}+6$ unknown parameters. According to Equations (3) to (7), there are 5 boundary equations at the upper and lower ends, 4 continuity equations at each node, and 1 residual equation at each span. There are altogether $5 \mathrm{~N}+6$ known equations. For the $\phi$ plane, the number of unknown parameters is same with known equations; thus the model is solvable. This model can use the bisection method to roughly select the existence range of the solution and then use Newton iteration to perform a fine search to obtain the final solution.

Using the solved coefficient $C_{k, i, j}$, the calculation results of the lateral force and slope on the bit can be obtained:

$$
\left\{\begin{array}{l}
F_{b, k}=6 E I_{1} C_{k, 2,1}+W_{b} C_{k, 4,1} \\
\theta_{b, k}=\arctan C_{k, 4,1} .
\end{array}\right.
$$

In Equation (8), $F_{b, k}$ is the component of the lateral force of the bit on the $\alpha$ and $\phi$ planes, $\theta_{b, k}$ is the component of the bit slope on the $\alpha$ and $\phi$ planes, and $W_{b}$ is the WOB.

Considering the buckling behavior of a tubular string under the influence of axial forces and bending moments, it is assumed that a is the proportional coefficient of the arbitrary span of the tubular string, and $e_{k}$ is the projection of the tubular string on the $\alpha$ and $\phi$ planes to the $z$-axis; when the borehole is eccentric distance, we can know the eccentric distance between the RSBHA and the borehole axis under the actual wellbore trajectory:

$$
G_{k, j}=\frac{\left[u_{k, j}\left(L_{k, j} / a\right)-e_{k}\right]}{\cos \psi_{k, j}^{\prime}} .
$$

Then, the mechanical behaviors of push-the-bit RSS can be solved with the above model.

2.2. Prediction Model of Drilling Penetration. The mechanical model of the RSS solves the mechanical load and the deflection angle of the bit during the actual drilling process. In this paper, the effect of formation properties is introduced into the conversion coefficient in Equation (14), which simplifies the calculation of anisotropic formation, and the known mechanical load on the bit and the bit slope can be substituted into the bit anisotropy model [14]. The prediction of the equation further takes into account the difference in cutting performance of the bit in different directions and the effect of the anisotropic formation being drilled:

$$
\left[\begin{array}{l}
R_{a} \\
R_{1} \\
R_{2}
\end{array}\right]=D_{n} \cdot \mathbf{S}^{T} \cdot\left[\begin{array}{lll}
1 & & \\
& I_{b} & \\
& & I_{b}
\end{array}\right] \cdot \mathbf{S} \cdot\left[\begin{array}{l}
W_{b} \\
F_{b, 1} \\
F_{b, 2}
\end{array}\right] .
$$

Among them, $R_{a}, R_{1}$, and $R_{2}$ are the components in the drilling penetration along the borehole axis direction, well inclination, and the lateral direction of azimuth plane, $D_{n}$ is the standard drilling efficiency, $S$ is the bottom hole coordinate and the bit coordinate conversion relationship, $I_{b}$ is the anisotropy index of the bit, and $W_{b}, F_{b, 1}$, and $F_{b, 2}$ are the mechanical load on the bit.

\section{Evaluation Methods of Build Rate}

3.1. Lateral Force Method. During the analysis of the prediction of the build rate, the lateral force of the bit can reflect the spatial shape of the trajectory most intuitively when the characterization of the formation parameters is not obvious. Theoretically, although the lateral force of the bit can be determined by a series of mechanical models, the magnitude of the lateral force is obtained through the inversion of the drilling data in actual drilling. In addition, the lateral force of the bit is not only determined by the mechanical force of the bit but also related to the parameters of the wellbore geometry [20,21], differences of the bit structure, formation anisotropy, and formation mechanical properties. At the same time, the lateral cutting capacity of the bit, the wear situation of the bit, and the expansion of the wellbore also greatly affect the prediction of the lateral force of the bit with the continuous adjustment of the tool face. Therefore, although this method is relatively mature in theory, it is not conducive to field application.

3.2. Inclination Trend Angle Method. The well inclination trend angle method considers the influence of the bit anisotropy and formation anisotropy. The angle $\theta_{t, k}$ between the direction of the resultant displacement and the bit axis is obtained via calculating the resultant displacement under the combined action of the lateral force of the bit and WOB. It explains the movement trend of the bit during the drilling. Due to an included angle $\theta_{b, k}$ between the bit axis and the wellbore axis, the final direction of the bit can be expressed as

$$
\gamma=\theta_{t, k}+\theta_{b, k},
$$

where $\gamma$ is the inclination angle of the bit direction from the wellbore axis. This method overcomes the problem that the lateral force method is not conducive to field application to some extent.

3.3. Ultimate Build Rate Method. In the former calculation of the build rate of the BHA, the DLS was considered to be the ultimate build rate when it was corresponding to zero lateral force at drill bit. However, the effects of bit anisotropy and formation anisotropy were neglected. The anisotropy between the bit and formation is closely related to the drilling trajectory; thus it cannot be ignored by the influence of the build rate and needs to be taken into account. Based on the previous studies, this article proposes two concepts about the build rate: 
Ultimate build rate: DLS while lateral drilling rate is zero.

Actual build rate: actual build rate during drilling.

The ultimate build rate refers to the limit condition reached in drilling process. This method comprehensively considers the inclination angle, the borehole diameter, structure of BHA, the bit anisotropy, WOB, the anisotropic formation, the direction and tendency of the formation, and other parameters. For the convenience of application, the influence of formation-related factors tends to be ignored. At this time, the ultimate build rate refers to the limit capacity of the drilling tool.

The actual build rate is not only related to the formation, drilling tools, and other factors but also closely related to the drilling process. A long process is required before reaching the ultimate build rate. However, parameters such as inclination angle are constantly changing during this process, so the ultimate build rate cannot be reached. In addition, the tool surface cannot be kept constant during the drilling process, so that the actual build rate is less than the ultimate build rate.

According to the definition of the ultimate build rate, its calculation model is as follows:

$$
\text { OBJ: }\left[\begin{array}{l}
R_{1}\left(\kappa_{1}\right) \\
R_{2}\left(\kappa_{2}\right)
\end{array}\right] \longrightarrow\left[\begin{array}{l}
0 \\
0
\end{array}\right] .
$$

Some researchers [17] use zero lateral force on drill bit to calculate the ultimate build rate; namely,

$$
\text { OBJ: }\left[\begin{array}{l}
F_{b, 1}\left(\kappa_{1}\right) \\
F_{b, 2}\left(\kappa_{2}\right)
\end{array}\right] \longrightarrow\left[\begin{array}{l}
0 \\
0
\end{array}\right] .
$$

Compared with Equation (12), the effects of bit-rock interaction are neglected in Equation (13).

Equation (12) solves the values of $\kappa_{1}$ and $\kappa_{2}$ by using Newton iteration method. The calculation formula for the ultimate build rate is expressed by the following equation:

$$
\kappa_{L}=\sqrt{\kappa_{1}^{2}+\kappa_{2}^{2}}
$$

The actual build rate cannot be directly solved theoretically, and inversion calculations need to be performed with the help of actual drilling data. However, the actual drilling data only contains the trajectory data of a specific drilling tool under specific conditions, it is difficult to reveal the influence of each factor on the build rate, and it cannot guide the optimal design of the drilling tool combination. Combining theoretical analysis and practical experience and introducing a conversion coefficient on the basis of the ultimate build rate, an approximate formula for the actual build rate can be obtained:

$$
\kappa_{A}=\lambda \cdot \kappa_{L} .
$$

The conversion factor is closely related to the drilling process, and its values range from 0.6 to 0.9 .

\section{Case Study}

Push-the-bit RSS has the characteristics of leverage effect, pendulum effect, and packed hole effect, which are possessed of the traditional steerable drilling tool. In the middle of them, the leverage effect has the features of build which is opposite to the pendulum effect, and the packed hole effect has played the function of a hold and drop. However, the pushing effect produced by hitting the wellbore is the most prominent feature of push-the-bit RSS. In order to reach the efficiency of building and dropping, the directional drilling engineer needs to control the magnitude and direction of the pushing force by computer.

4.1. Comparison of Different Methods. Table 1 shows the drilling tool parameters of push-the-bit RSS, including drill bit, orient head, the flexible sub, gamma, MWD, stabilizer, BCPM, collar, and drill pipe. The conversion coefficient of the build rate $\lambda=0.75$.

Figure 3 shows the distribution of the lateral force, trend angle, and ultimate build rate when the inclination angle changes under the specified conditions, of which the pushing force is $2 \mathrm{t}$, WOB is $8 \mathrm{t}$, bit anisotropy is 0.07 , mud density is $1.5 \mathrm{~g} / \mathrm{cm}^{3}$.

The results of lateral force and trend angle indicate that the build rate first increases and then decreases with the increase of inclination angle. However, the results of the ultimate build-rate method indicate that the build rate first decreases, second increases, and then slowly decreases with the increase of inclination angle. The reasons for the differences among these three methods are as follows:

(1) Lateral force method: this method only uses lateral force to represent build rate but does not take into account deviation angle between bit axis and wellbore axis, bit-rock interaction, and so on.

(2) Trend angle method: this method uses deviation angle between bit axis and wellbore axis to represent build rate, but the trend angle is different from build rate and quantitative relation between trend angle and build rate is unknown.

(3) Ultimate build-rate method: kinds of factors are sufficiently considered in this method, but the value of correction factor should be calculated with drilling data. Therefore, the value of the build rate is generally higher than the practical build rate.

4.2. Effect of Pushing Force Magnitude and Orientation. Figure 4 shows the build rate of the pushing block in different directions. When the pushing block is fully retracted $\left(F_{p}=0 \mathrm{KN}\right)$, the build rate of push-the-bit RSS does not change with the change of the direction of the pushing block, which means that the RSS is not affected by pushing effect. When the pushing force is not 0, push-the-bit RSS will be affected by the pushing effect. As the pushing force is 
TABle 1: Parameters of push-the-bit RSS.

\begin{tabular}{lcc}
\hline Name & Diameter (in.) & Length (ft) \\
\hline Bit & 8.5 & 1.312 \\
Offset mechanism & 8.188 & 0.337 \\
Stabilizer & 8.366 & 0.492 \\
Flexible sub & 5 & 3.143 \\
Gamma & 7.244 & 3.478 \\
MWD & 7 & 10.33 \\
BCPM & 7.244 & 3.396 \\
Collar & 7 & 121.391 \\
Drilling string & 5 & 6561.679 \\
\hline
\end{tabular}

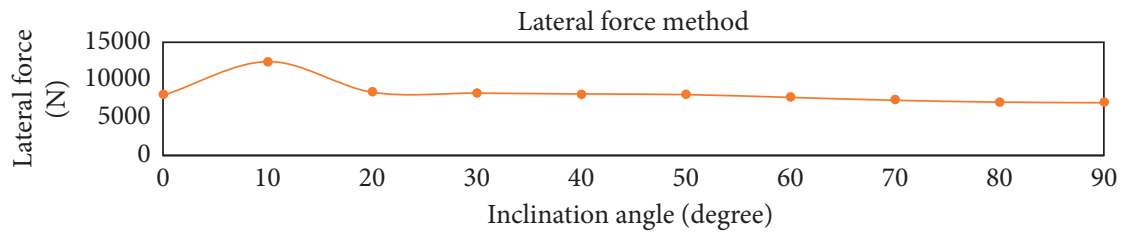

(a)

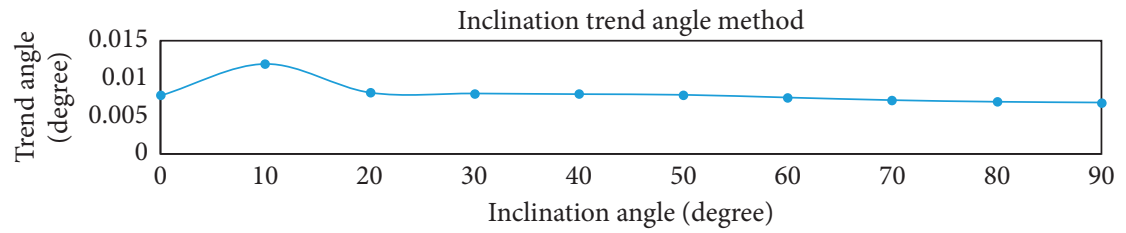

(b)

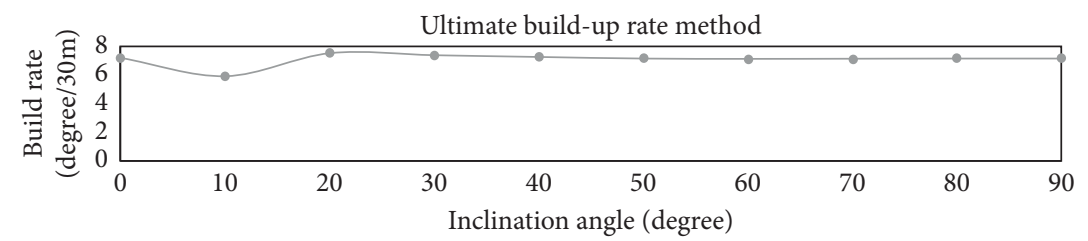

(c)

Figure 3: Evaluation results of tool build rate.

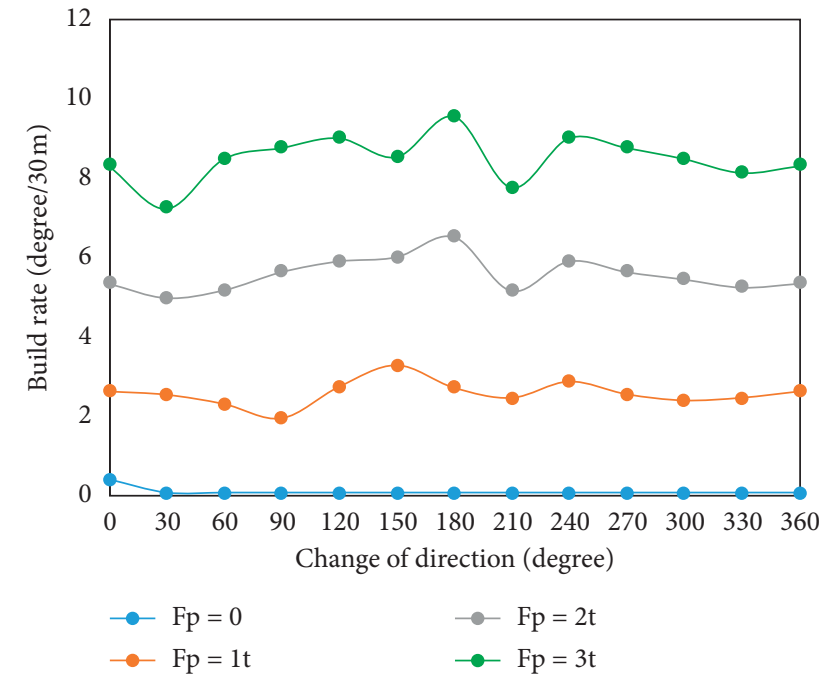

FIgUre 4: Build rate of the pushing block in different directions. increased, the build rate of the steerable tool during the drilling process will increase for the pushing effect increases. However, this plot also shows the fluctuation of the pushing force, indicating that when the pushing block is situated in different borehole positions, the pushing coefficient will cause the RSS to build and drop the working conditions.

4.3. Effect of Inclination Angle. Figure 5 shows the build rate under different pushing forces and inclination angles when WOB is $8 \mathrm{t}$. The increase of the build rate is approximately positive correlation between the magnitudes of the pushing force, which indicates that the pushing effect plays an essential role in the building process. With the increase of the inclination of the well, the pendulum effect will gradually weaken and the leverage effect will gradually increase. Therefore, the build rate will decrease first and then increase.

4.4. Effect of WOB. Figure 6 reveals that the condition of WOB increases; the overall tendency of the build rate will decrease. This is because as WOB increases, the ratio of the 


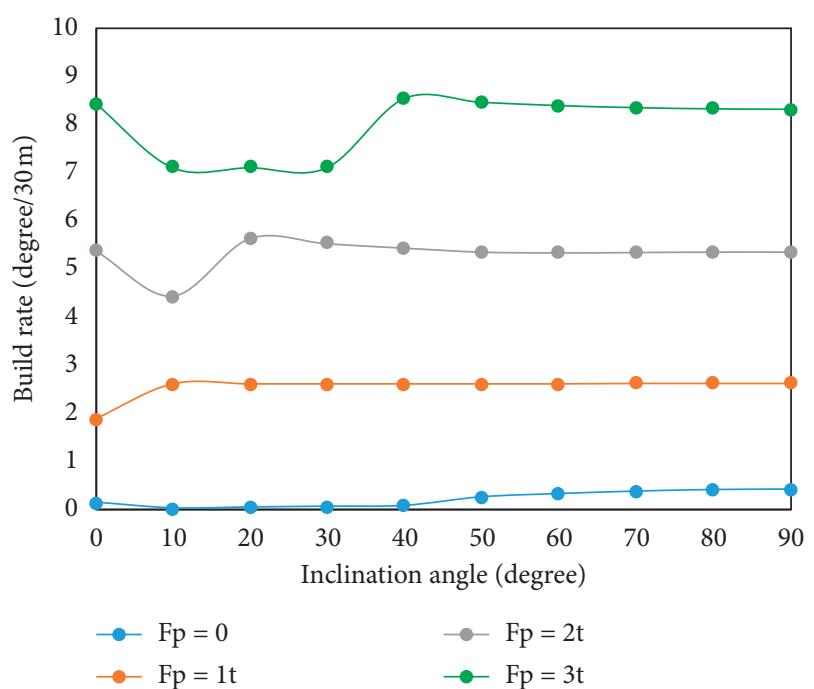

FIGURE 5: Build rate under different pushing forces and inclination angles.

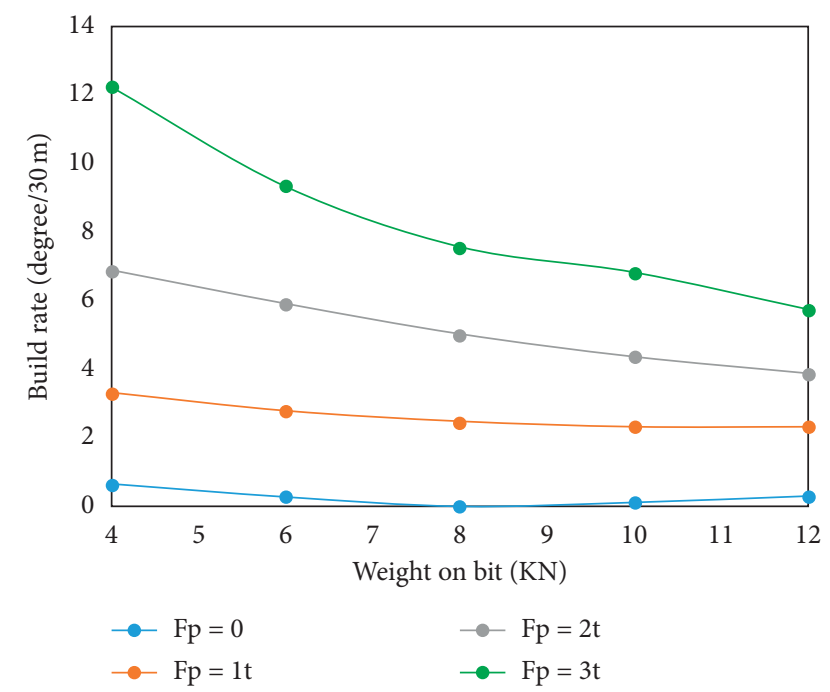

Figure 6: Build rate under different pushing forces and weights on bit.

pushing force to WOB decreases, and the relative function of the pushing force on the drilling trajectory decreases; that is, the pushing effect decreases.

4.5. Effect of Well Diameter Expansion. Figure 7 considers the possibility of well diameter expansion during building process. In the case of no the pushing force, with the borehole diameter being enlarged, the build rate decreases before it increases. When the pushing force is not zero, the expansion of the well diameter will slightly increase in the change of the build rate. It is noted that excessively high pushing force will cause fluctuations in the build rate. This situation is not only the result of the comprehensive effects of pushing effect, leverage effect, pendulum effect, and so on,

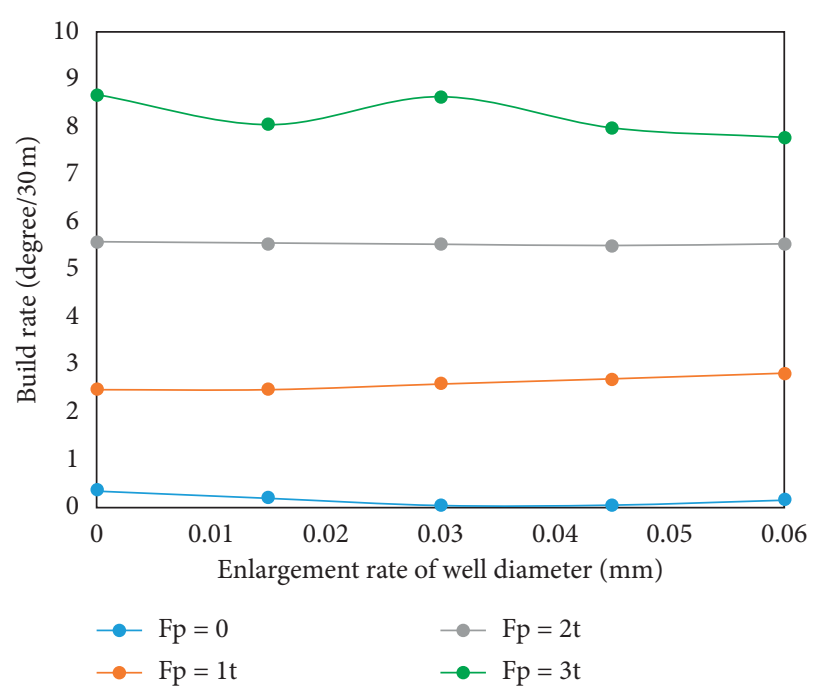

FIGURE 7: Well diameter expansion during the (building) kicking off.

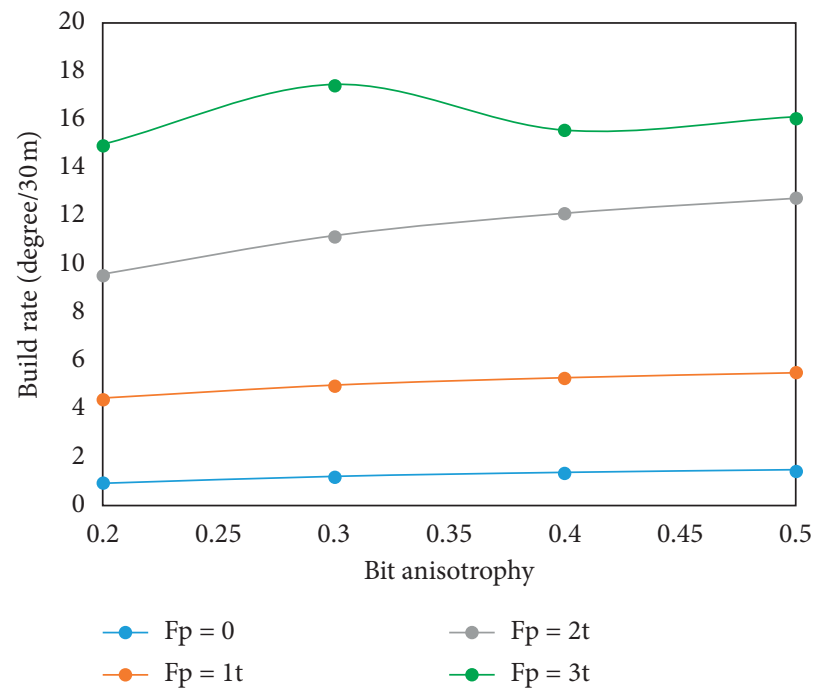

FIGURE 8: Build rate under different pushing forces and bit anisotropy index.

but also the cutting property of drill bits, anisotropy formation, and other factors.

4.6. Effect of Bit Anisotropy. Figure 8 shows the effect of bit anisotropy index $\left(I_{b}\right)$ on build rate. With the increase of $I_{b}$, the cutting ability of the drill is continuously improved. When $I_{b}=1$, it is an isotropic bit. The lateral cutting ability is the same as the axial cutting ability. When $I_{b}=0$, the bit has only axial cutting ability and no lateral cutting ability. The mechanism of the pushing effect is to boost the lateral force of the bit. If the cutting load is too large, the area of the rock broken along the shear plane will increase, and it leads to the pendulum effect due to the expansion of the well diameter. Therefore, when $F_{p}=3 \mathrm{t}$ in the plot, the build slope will be a slight decline. 


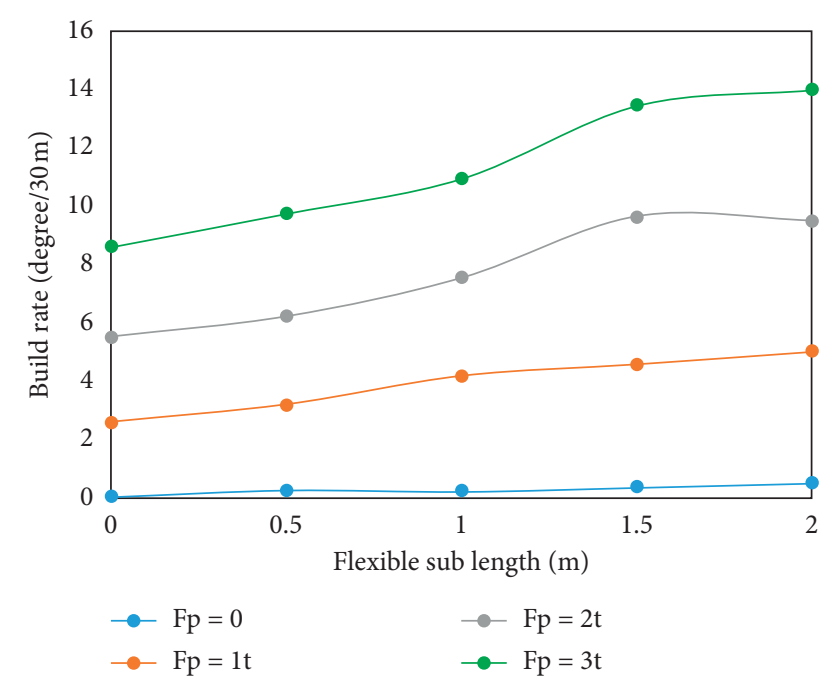

FIgURE 9: Buckling the influence of the length of the flexible sub for the build rate.

4.7. Effect of Tool Structure. Figure 9 reveals the influence of the structure of push-the-bit RSS on the build rate by changing the length of the flexible sub. As the length of the flexible sub increases, the build rate also increases. Since the stiffness of the flexible sub is apparently smaller than the stiffness of the adjacent pipe string, the influence of the build-rate capabilities can be reduced by decreasing the stiffness of the flexible sub. With the length of the flexible sub increasing, the influence of the rigidity of the upper drilling tool decreases, which is more conducive to the exertion of the leverage effect.

\section{Conclusions}

(1) Compared with the lateral force method and the trend angle method, the new method can calculate build rate directly. Compared with ultimate buildrate method based on zero lateral force, the new method further considers the effects of bit cutting performance and formation anisotropy with zero lateral drilling penetration criterion and extends two-dimensional case to three-dimensional cases. Therefore, the new method provides a more accurate calculation method of the build rate of push-the-bit RSS.

(2) The build rate of push-the-bit RSS mainly depends on the combined results of "leverage effect," "pendulum effect," and "pushing effect." The pushing effect has the most significant effect on the steerable tool build rate, so the correlation is the highest between the drilling tool build rate and the pushing force. The leverage effect and the pendulum effect play the secondary role; thus the influence of the inclination angle and the WOB on the build rate is relatively less than the influence of push force on the build rate.

(3) For conventional sliding steerable drilling tools, as WOB increases, the leverage effect increases, and the build rate also increases; for push-the-bit RSS, as WOB increases, the proportion of leverage effect increases, and the proportion of the pushing effect decreases, so the build rate actually goes down. The existence of the pushing effect makes the building mechanism of the push-the-bit RSS more complicated than the conventional sliding steerable drilling tool, and even the opposite influence regularity can be occurred.

(4) Build rates are closely related to tool structure parameters (size and length of the drill string, the length of flexible sub, etc.), pushing force (size and direction), performance of the drill bit (anisotropic index), drilling parameters (WOB, etc.), and formation properties (strike and dip angles, anisotropy index, etc.). In order to control the wellbore trajectory more precisely, it is indispensable to design of tool structure and pushing force magnitude, and an accurate understanding of the nature of the formation is also a vital step when it needs to be drilled. Finally, the drilling parameters and push force should be controlled reasonably.

(5) The ultimate build rate is an ideal result. The actual build rate is generally smaller than the ultimate build rate. There is an approximate proportional relationship between them. The proportional coefficient is closely related to the drilling process. Particularly, when formation anisotropy is considered, it is more difficult to confirm the proportionality coefficient. The next step is to study how to determine the coefficient reasonably.

\section{Nomenclature}

RSS: $\quad$ Rotary steerable system

BHA: Bottom-hole assembly

$u_{k}$ : Deflection of the tubular string on the inclination plane and azimuth plane $(\mathrm{m})$

$q_{k}$ : $\quad$ Line weight of the tubular string on the inclination plane and azimuth plane $\left(\mathrm{kN} \cdot \mathrm{m}^{-1}\right)$

EI: $\quad$ Bending stiffness of the tubular string $\left(\mathrm{N} \cdot \mathrm{m}^{2}\right)$

$F$ : $\quad$ Axial force on the tubular string (N)

$L_{k, N+1}$ : Length of upper tangential contact point (m)

$r_{N+1}$ : Apparent radius of wellbore at upper tangential contact point (mm)

$\psi_{k, N+1}$ : Projection of the DLS from the bit to the upper tangential contact point on the inclination and azimuth plane (rad)

$L_{k, j}: \quad$ Length of the $j$ node $(\mathrm{m})$

$r_{j}: \quad$ Apparent radius of wellbore at the position of the $j$ node $(\mathrm{mm})$

$\psi_{k, j}$ : $\quad$ Projection of the DLS from the bit to the node current on the inclination and azimuth plane (rad)

$F_{k, p}$ : Component of pushing force projected on inclination plane and azimuth plane $(\mathrm{kN})$

$F_{b, k}$ : Component of the lateral force of the bit on inclination plane and azimuth plane $(\mathrm{kN})$ 
$\theta_{b, k}: \quad$ Component of the bit slope on inclination plane and azimuth plane (rad)

$e_{k}$ : $\quad$ Projection of the tubular string on inclination and azimuth plane to the $z$-axis when the borehole is eccentric distance $(\mathrm{m})$

$\psi_{k, j}{ }^{\prime}: \quad$ The deviation rate of tubular string at $L_{k, j} / a$ $\left(\right.$ rad. $\left.\mathrm{m}^{-1}\right)$

$G_{k, j}$ : $\quad$ Eccentric distance between the RSBHA and the borehole axis under the actual wellbore trajectory (m)

$W_{b}: \quad$ Weight on bit $(\mathrm{kN})$

$R_{a}, R_{1}$, Components in the drilling penetration along the

$R_{2}$ : $\quad$ borehole axis direction, well inclination, and the lateral direction of azimuth plane $\left(\mathrm{m} \cdot \mathrm{h}^{-1}\right)$

$\theta_{t, k}: \quad$ Angle between combined displacement direction and bit axis (rad)

$\gamma$ : Inclination angle of the bit direction from the wellbore axis (rad)

$\kappa_{L}: \quad$ Ultimate build rate projected $\left(\mathrm{m}^{-1}\right)$

$\kappa_{1}, \kappa_{2}$ : Ultimate build rate projected on inclination plane and azimuth plane $\left(\mathrm{m}^{-1}\right)$

$K_{A}: \quad$ Actual build rate $\left(\mathrm{m}^{-1}\right)$

$F_{p}: \quad$ Resultant of pushing force $(\mathrm{kN})$.

\section{Data Availability}

The data used to support the findings of this study are included within the article.

\section{Conflicts of Interest}

The authors declare that they have no conflicts of interest.

\section{Acknowledgments}

The authors gratefully acknowledge the financial support from the Natural Science Foundation of China (Grants nos. 51821092, U1762214, and 51904317) and Science Foundation of China University of Petroleum, Beijing (Grant no. ZX20180414). This research was also supported by other projects (Grants nos. 2017ZX05009-003 and 2016YFC0303303).

\section{References}

[1] D. Gao, Optimized Design and Control Techniques for Drilling \& Completion of Complex-Structure Wells, China University of Petroleum Press, Dongying, China, 2011.

[2] W. Hu, "The present and future of low permeability oil and gas in China," China National Petroleum Corporation, vol. 11, no. 8, pp. 29-37, 2009.

[3] W. Jiang, S. Jiang, L. Sheng, X. Fu, and P. Chen, "Research on rotary navigation drilling tools and its application," Oil Drilling \& Production Technology, vol. 5, pp. 21-24, 2008.

[4] W. Jiang, S. Jiang, X. Fu et al., "Application of rotary steering drilling technology and its research progress," Natural Gas Industry, vol. 33, no. 4, pp. 75-79, 2013.

[5] X. Zhu, Q. Liu, H. Li, and H. Tong, "Study on theory and method of borehole trajectory prediction," Natural Gas Industry, vol. 4, pp. 38-40, 2004.

[6] H. Jerez and J. Tilley, "Advancements in powered rotary steerable technologies result in record-breaking runs," in
Proceedings of the SPE Latin America and Caribbean Petroleum Engineering Conference, Maracaibo, Venezuela, May 2014.

[7] A. A. Al Dabyah, B. A. Zubairi, I. S. Al Umairin et al., "Proper planning supported by latest technology leads to drilling the longest 8. 5 in section in an extended-reach well: case study," in Proceedings of the Offshore Technology Conference Asia, Kuala Lumpur, Malaysia, March 2016.

[8] P. Wang, L. Sheng, X. Dou et al., "Ne-west development and tendency of foreign rotary steerable systems," Drilling and Production Technology, vol. 36, no. 6, pp. 32-35, 2013.

[9] H. Karisson, T. Brassfield, and V. Kruger, "Performance drilling optimization," in Proceedings of the SPE/IADC Drilling Conference, New Orleans, Louisiana, March 1985.

[10] W. B. Bradley, "Factors affecting the control of borehole angle in straight and directional wells," Journal of Petroleum Technology, vol. 27, no. 6, 1975.

[11] Q. Di and G. Peng, "A new method for predicting the deflecting capacity of bottom hole assembly," Oil Drilling \& Production Technology, vol. 2, pp. 17-19+81, 2000.

[12] D. Qingfeng, "Analysis of effect of bottom hole assembly on whipstocking capacity," Petroleum Drilling Techniques, vol. 1, pp. 39-41, 2000.

[13] Y. Xia, Z. Guan, and Y. Shi, "Evaluation method of parameters design for bottom hole assembly," Journal of the University of Petroleum, China, vol. 29, no. 4, pp. 48-51, 2005.

[14] D. Gao, C. Nie, and X. Liu, "A Study on anisotropic drilling characteristic of drilling bit," Journal of the University of Petroleum, China, vol. 6, pp. 28-31, 1991.

[15] D. Zheng, D. Gao, J. Feng, and H. Zhang, "Study on deflection performance of backup and directional types of rotary steering tools," Oil Drilling \& Production Technology, vol. 33, no. 6, pp. 10-13, 2011.

[16] D. Zheng, H. Zhang, Y. Zhu et al., "Affecting mechanism of rock mechanical property on well trajectory," Petroleum Drilling Techniques, vol. 3, pp. 45-49, 2014.

[17] Y. Su, "A method of limiting curvature and its applications," Journal of Petroleum, vol. 3, pp. 112-116, 1997.

[18] D. Gao, X. Liu, and B. Xu, Prediction and Control of Wellbore Trajectory, China University of Petroleum Press, Dongying, China, 1994.

[19] J. Bai and Y. Su, Theory and Practice of Well Deviation Control, Petroleum Industry Press, Beijing, China, 1990.

[20] J. Sugiura, "Optimal BHA design for steerability and stability with configuration rotary steerable system," in Proceedings of the SPE Asia Pacific Oil and Gas Conference and Exhibition, Perth, Australia, October 2008.

[21] Z. Han, "New technology of the design and control of the 3dimension trajectory," Petroleum Drilling Techniques, vol. 31, no. 5, p. 1, 2003. 